\title{
Guest Editor's Note: High-Level Parallel Programming 2019
}

\section{Christoph Kessler ${ }^{1}$}

Published online: 15 July 2020

(C) Springer Science+Business Media, LLC, part of Springer Nature 2020

This special issue contains revised versions of selected papers from the 12th International Symposium on High-Level Parallel Programming and Applications (HLPP-2019), held 3-5 July 2019 in Linköping, Sweden.

The HLPP series of workshops/symposia, started in 2001, is a forum for researchers developing state-of-the-art concepts, tools and applications for highlevel parallel programming. The general emphasis is on software quality, programming productivity and high-level performance models.

After a rigorous peer-review process in two stages performed by the HLPP-2019 program committee members and additional reviewers (in a first stage for presentation at the HLPP-2019 symposium and in a second stage for inclusion in the special issue), revised versions of 7 of the papers presented at HLPP-2019 have been accepted for publication in this special issue:

- Christopher Brown, Vladimir Janjic, Adam Barwell, Jose Daniel Garcia, Kenneth MacKenzie: Refactoring GrPPI: Generic Refactoring for Generic Parallelism in $\mathrm{C}++$

- Christopher Brown, Vladimir Janjic, Mehdi Golo, John McCall: Programming Heterogeneous Parallel Machines using Refactoring and Monte-Carlo Tree Search

- Frédéric Gava, Yoann Marquer: Axiomatization and Imperative Characterization of Multi-BSP Algorithms

- Clemens Grelck, Cedric Blom: Resource-aware Data Parallel Array Processing

- Marcel Köster, Julian Groß, Antonio Krüger: Massively Parallel Rule-Based Interpreter Execution on GPUs using Thread Compaction

- Luca Rinaldi, Massimo Torquati, Daniele De Sensi, Gabriele Mencagli, Marco Danelutto: Improving the Performance of Actors on Multi-Cores with Parallel Patterns

- Fabian Wrede, Herbert Kuchen: Towards High-Performance Code Generation for Multi-GPU Clusters Based on a Domain-Specific Language for Algorithmic Skeletons

Christoph Kessler

Christoph.Kessler@liu.se

$1 \quad$ Linköping University, Linköping, Sweden 
I would like to thank all members of the HLPP-2019 program committee: Marco Aldinucci (University of Torino, Italy), Ioana Banicescu (Mississippi State University, USA), Jost Berthold (Digital Asset, Australia), Murray Cole (University of Edinburgh, UK), Frederic Dabrowski (LIFO/Univ. Orleans, France), Rudolf Eigenmann (University of Delaware, USA), Franz Franchetti (Carnegie Mellon University, USA), Jose Daniel Garcia (Univ. Carlos III Madrid, Spain), Frederic Gava (Univ. Paris-East, France), Alex Gerbessiotis (New Jersey Inst. Techn., USA), Arturo Gonzalez-Escribano (Univ. Valladolid, Spain), Sergei Gorlatch (Univ. of Münster, Germany), Clemens Grelck (Univ. Amsterdam, Netherlands), Dalvan Griebler (PUCRS/SETREM, Brasil), Gaetan Hains (Huawei Paris Research Center, France), Ludovic Henrio (CNRS Lyon, France), Peter Kilpatrick (Queen's Univ. Belfast, UK), Herbert Kuchen (Univ. of Münster, Germany), Frederic Loulergue (Northern Arizona University, USA), Kiminori Matsuzaki (Kochi Univ. of Technology, Japan), Aleksandar Prokopec (EPFL Lausanne, Switzerland), Kostis Sagonas (Uppsala University, Sweden), Michel Steuwer (Univ. Glasgow, UK), Massimo Torquati (Univ. Pisa, Italy), Jesper L. Träff (Vienna Univ. of Technology, Austria), Ana Lucia Varbanescu (Univ. Amsterdam, Netherlands).

I also thank the additional reviewers for HLPP-2019 and for the special issue: Parinaz Barakhshan, Akshay Bhosale, Christopher Brown, Iacopo Colonnelli, Florian Fey, Bastian Köpcke, Marius Kühnemund, Johan Lilius, Suejb Memeti, Breno Menezes, Thibaut Tachon, Maxwell Young.

Moreover, I thank the members of my group at Linköping University, in particular Suejb Memeti who co-organized HLPP-2019 with me, August Ernstsson for giving the tutorial on High-level portable programming of heterogeneous systems in SkePU, and Lene Rosell who helped with the administrative work.

Thanks also for the two excellent keynotes at HLPP-2019, by Georgi Gaydadjiev (Maxeler) on High-level programming of data-centric reconfigurable dataflow systems and Jose-Daniel Garcia (UC3M) on Generic Programming and Parallel Patterns.

Finally, I thank Lavanya Ravi from Springer for the good cooperation with the special issue management for IJPP.

Linköping, June 2020

Christoph Kessler, guest editor 